\title{
National survey of the surgical management of subfertility associated with uterine fibroids which do not distort the uterine cavity
}

\author{
G. Ahmad • J. M. N. Duffy • Y. Mohammed • \\ A. J. S. Watson
}

Received: 14 August 2008 / Accepted: 15 October 2008 / Published online: 15 November 2008

(C) Springer-Verlag 2008

\begin{abstract}
Uterine fibroids which do not distort the uterine cavity are associated with otherwise unexplained subfertility. There is currently no consensus as to their surgical management. Surgical intervention is left to the discretion of the treating gynaecologist. An anonymous postal questionnaire was sent to gynaecologists in the UK with an interest in subfertility to survey current clinical practice. At total of 245 questionnaires were sent out, and 133 (54\%) responses were analysed. Over a third of respondents would not intervene to remove a fibroid under any of the specified circumstances. Of the gynaecologists who would intervene, respondents were most likely to intervene in women aged between 30 and 40 years (43\%), at a uterine site (36\%), sized over $5 \mathrm{~cm}(10 \%)$ and with only one fibroid being present (14\%). Without further guidelines and high quality research, it remains difficult to determine the best treatment for these patients.
\end{abstract}

\footnotetext{
G. Ahmad

Department of Obstetrics and Gynaecology,

Stepping Hill General Hospital,

Manchester, UK

J. M. N. Duffy

Department of General Surgery, Guy's and St. Thomas' Hospital, London, UK
}

Y. Mohammed • A. J. S. Watson

Department of Obstetrics and Gynaecology,

Tameside General Hospital,

Manchester, UK

\author{
A. J. S. Watson $(\triangle)$ \\ Tameside General Hospital, \\ Fountain Street, \\ Ashton-under-Lyne, Lancashire OL6 9RW, UK \\ e-mail: andy.watson@tgh.nhs.uk
}

Keywords Uterine fibroids · Laparoscopic surgery Laparotomy · Subfertility

\section{Introduction}

Uterine fibroids are the most common neoplasms of the female genital tract. It is estimated that $50 \%$ of women of reproductive age will have one or more uterine fibroids [2]. Since the majority of women with fibroids are fertile, it is clear that the presence of fibroids does necessary impair fertility. It is estimated that fibroids are the sole cause of subfertility in $2-3 \%$ of subfertile women [2].

There are various possible mechanisms by which fibroids could result in subfertility. A popular possible mechanism involves the distortion of the uterine anatomy by a fibroid resulting in reduced implantation rates. For example, a fibroid located near the internal os of the cervix may prevent sperm migrating into the uterine cavity and the fallopian tubes. Therefore, it is thought that by restoring the normal anatomy of the uterine cavity by surgical intervention, there will be an improvement in any associated subfertility. A minority of fibroids do not distort the uterine anatomy; therefore, other mechanisms must exist which cause subfertility. It has been suggested that the endometrium overlying a fibroid may have reduced vascularity, which could lead to a failure of implantation. The literature also reports local inflammatory changes, increased vasoactive amines and androgens within the endometrial cavity which may achieve a similar effect [2]. It is also thought that fibroids may cause dysfunctional uterine contractility and could impact on sperm migration. Presently, there is no consensus in the literature as to how to manage fibroids which do not distort the uterine anatomy. However, there is some limited evidence that intramural fibroids do have an 
unfavourable effect on implantation rates during in vitro fertilisation treatment if they measure over $5 \mathrm{~cm}[1,3]$. Surgical intervention is left to the discretion of the treating gynaecologist.

Deciding when and which surgical intervention to perform proves a challenging management choice for a gynaecologist. Characteristics of the fibroids that may affect the gynaecologist's decision to surgically intervene include its site, size and the number of uterine fibroids present. We set out to survey current clinical practice within the UK.

\section{Materials and methods}

A list of gynaecologists was derived by an Internet search. Each healthcare trust's website was searched using keywords, for example subfertility and reproductive medicine. Trainees and specialist nurses were excluded. An anonymous postal questionnaire was sent to the identified parties (questionnaire available on request from the corresponding author).

The questionnaire consisted of a clinical scenario involving a nulliparous woman with primary subfertility with normal investigations with the exception of intramural fibroids which were not distorting the uterine cavity. This scenario promoted responses from the respondent with regards to the characteristics of the patient and fibroids that may affect their decision to surgically intervene. These were the patient's age, the site, size and number of fibroids present. A self-addressed envelope was provided for the questionnaire to be returned. Non-respondents were not followed up. The data were recorded electronically using Microsoft Excel.

\section{Results}

A total of 245 questionnaires were sent and 133 (54\%) were returned. No responses were excluded. Approximately a third of respondents would not intervene regardless of patient age (35\%), site $(37 \%)$, size $(30 \%)$ and the number of fibroids $(43 \%)$. A significant portion of respondents reported no view with regards to these characteristics. Of the respondents who would perform surgery, they reported a wide variation in practice with regards to how to select patients suitable for surgery based on patient age and the selected characteristics of the fibroids present (number, size and location; Fig. 1a-d).

The open myomectomy technique was favoured by 109 $(82 \%)$ respondents. The alternative method of laparoscopic myomectomy was favoured by $13(9 \%)$ respondents, all of whom had over 10 years of experience in laparoscopic surgery. Respondents stating no preference numbered 13 $(9 \%)$. The number of gynaecologists with over 10 years of experience of laparoscopic surgery numbered $110(83 \%)$. Respondents with less than 5 years of experience numbered three (2\%), with between 5 and 10 years of experience numbered $16(12 \%)$, and four $(3 \%)$ respondents made no indication of their level of experience.

\section{Discussion}

There is no consensus in the literature with regards to the management of uterine fibroids that do not distort the uterine cavity and are associated with subfertility. A questionnaire survey enabled the current clinical practice of gynaecologists nationwide to be expressed and analysed to determine whether a consensus in clinical practice can be established. As far as we are aware, this is the first survey of clinical practice in the UK addressing this clinical dilemma. The results demonstrate a mixed clinical practice in terms of whether a surgical intervention is favoured, what surgical technique is favoured and which fibroid and patient characteristics would influence their decision.

A limitation of our survey involved the description of a standardised patient within the questionnaire to stimulate the respondent. Clearly, in everyday practice, few patients are typical and the unique individual clinical circumstances of each patient will influence management. Another limitation of our survey was that only a few carefully selected fibroid and patient circumstances, for example patient age, were enquired about. Responding gynaecologists would be likely to take into account a more extensive set of circumstances, for example patient wishes, suitability for surgery, bleeding abnormalities and the rate of fibroid growth, when deciding upon management. The survey would have also benefited from asking the respondents how long they would wait before offering myomectomy.

Over a third of respondents would not surgically intervene to remove a fibroid that did not distort the uterine cavity and were associated with subfertility regardless of patient age $(35 \%)$, site $(37 \%)$, size $(30 \%)$ and the number of fibroids $(43 \%)$. This may reflect a traditional view of gynaecologists not to intervene surgically when managing fibroids associated with subfertility. This is consistent with reports in the literature; in one series of 677 fertility enhancing procedures, it was reported that fibroids were the sole indication for surgery in $2.4 \%$ of cases [2]. This opinion may also reflect the sparsity of evidence within the literature; no casual link between uterine fibroids and subfertility has yet been established.

Despite $110(83 \%)$ respondents having ten or more years experience of laparoscopic surgery, $109(82 \%)$ respondents preferred an open method instead of a laparoscopic 

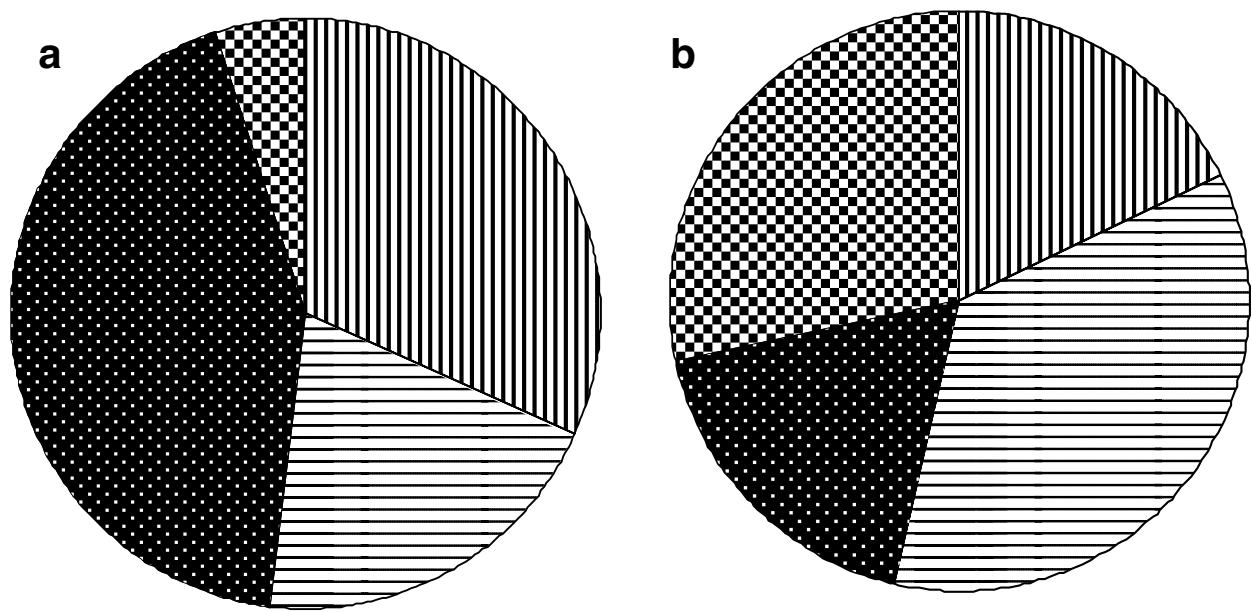

$$
\begin{array}{ll}
\text { m No opinion } & \text { 目 }<30 \text { years old } \\
\text { m 30-40 years old } & \text { ⿴囗十 }>40 \text { years old }
\end{array}
$$
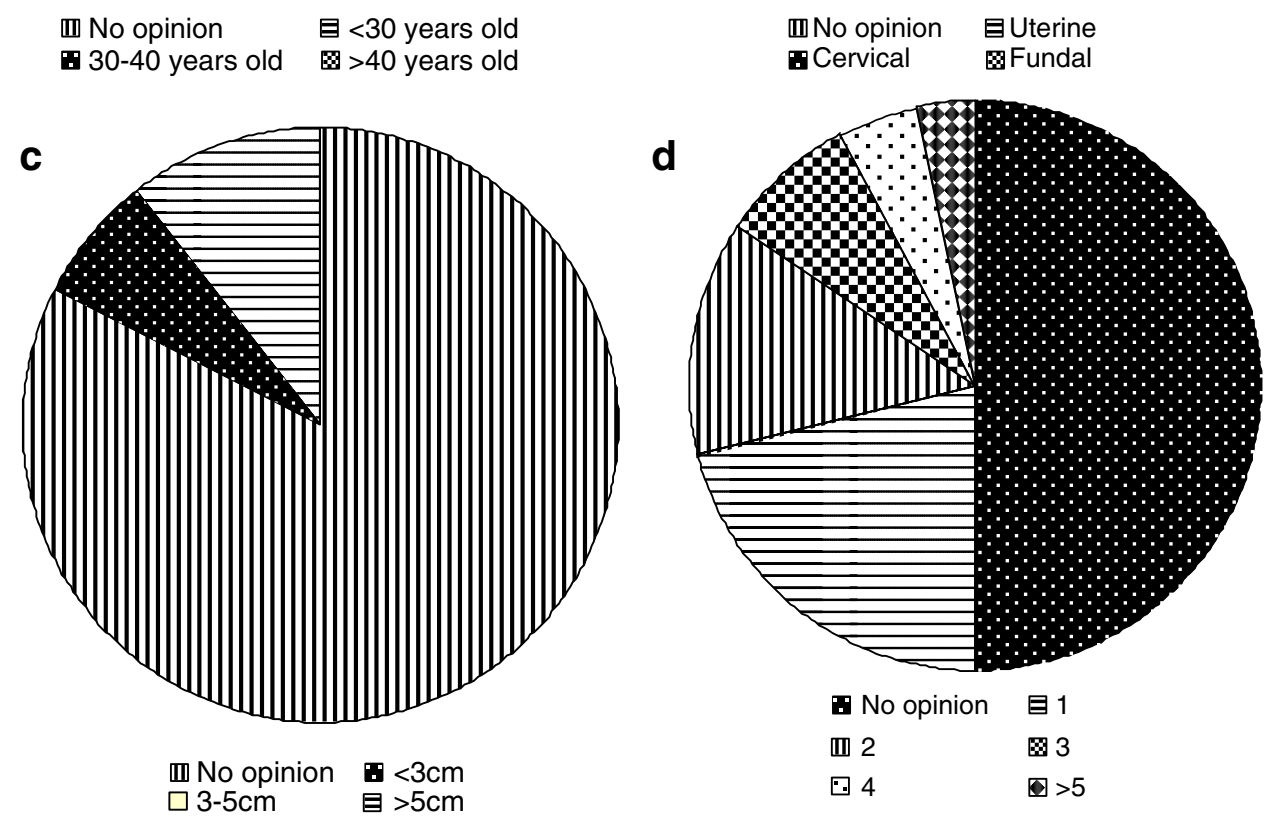

Fig. 1 a Patient's age. Of the respondents who would operate to remove a fibroid, no age preference was indicated by $31(32 \%)$ respondents. Women aged under 30 years were preferred by $20(20 \%)$ respondents, women aged between 30 and 40 years were preferred by $42(43 \%)$ respondents and five $(5 \%)$ respondents stated a preference to operate on women aged over 40 years. b Site of fibroid. Of the respondents who would operate to remove a fibroid, no site preference was indicated by $17(17 \%)$ respondents. A uterine site was favoured by $34(36 \%)$ respondents, a cervical site by $17(17 \%)$ respondents and a fundal site by $27(30 \%)$ respondents. c Size of fibroid. Of the respondents who would operate to remove a fibroid, no size

preference was indicated by $71(83 \%)$ respondents. A fibroid measuring less than $3 \mathrm{~cm}$ was favoured by six (7\%) respondents, a fibroid measuring 3 to $5 \mathrm{~cm}$ was favoured by no respondents and a fibroid measuring over $5 \mathrm{~cm}$ was favoured by nine $(10 \%)$ respondents. d Number of fibroids. Of the respondents who would operate to remove a fibroid, no number preference was indicated by $45(50 \%)$ respondents. One or more fibroids would be removed by $19(22 \%)$ respondents, two or more fibroids would be removed by $12(14 \%)$ respondents, three or more fibroids would be removed by seven $(7 \%)$ respondents, four or more fibroids would be removed by four $(4 \%)$ respondents, and five or more fibroids would be removed by three $(3 \%)$ respondents

technique in performing a myomectomy. Several studies have reported improved pregnancy rates ranging between $50 \%$ and $68 \%$ following abdominal and laparoscopic myomectomy when compared to a control group [2]. Few studies have directly compared open and laparoscopic myomectomy. A review of laparoscopic myomectomy concluded that it is a preferable procedure compared to an open technique. The study concluded that with similar improvements in subfertility, the operative and postoperative morbidity associated with either procedure must also be taken into account. Laparoscopic myomectomy offers shorter hospitalisation, faster recovery, fewer adhesions, less postoperative pain, a reduced risk of haemorrhage and minimal aesthetic damage compared with the open technique. However, concern has been expressed regarding the integrity of the scar and its ability to withstand labour [6]. 
Another risk which needs careful consideration is the risk of conversion to laparotomy during laparoscopic surgery. According to the logistic regression analysis Dubuisson performed, even in skilled hands, the size, type and location of the fibroid are all independent risk factors for conversion [4]. A recent review of the literature also suggests that when more than two fibroids with a total uterine size in excess of 12 weeks, few gynaecologists have the skills to remove these fibroids laparoscopically [5].

A minority of respondents (23\%) would intervene when considering women aged less than 30 years. It is reasonable to assume that most gynaecologists would deploy watchful waiting in women of this age group so as to provide an opportunity for the women to achieve spontaneous conception. The difficulties of counselling individuals in this age group about the risks and benefits of interventions are problematic, as no specific risk profiles exist in the literature. Respondents (32\%) are more likely to intervene in women aged between 30 and 40 years. They may be encouraged by the literature reporting improved pregnancy rates following abdominal and laparoscopic myomectomy in this age group. Respondents (4\%) were least likely to intervene when considering women aged over 40 . There is a lack of evidence in the literature demonstrating any benefit of intervention in this age group. However, a study has demonstrated an improvement in implantation rates during in vitro fertilization cycles when fibroids are removed in this age group [7]. Gynaecologists may also be wary to intervene due to significant obstetric complications of women aged over 40 years.

Of the respondents who would intervene, when considering the site of a fibroid, a uterine site was most favoured (34\%), followed by a fundal site $(17 \%)$ and then by a cervical site (17\%). As far as we are aware, no studies have investigated the potential benefit, in terms of improved fertility, of surgical interventions directly comparing different fibroid locations which do not distort the uterine cavity. The literature suggests that a fibroid located near the internal os of the cervix may prevent the migration of sperm into the uterine cavity and fallopian tubes. In addition, a fibroid could cause distortion of the fallopian tube if located near the uterine cornus [2].

Respondents who would intervene, favoured an intervention as the size of the fibroid increased. Of the studies which have reported improved fertility outcomes with surgical removal of the fibroids, the mean fibroid size has been measured in both the treatment and control groups. Consistently, when the mean size of fibroid is over $3 \mathrm{~cm}$ in the treatment group, estimated by transvaginal sonography, fertility improved when these fibroids were removed when compared to control groups [2]. It remains unclear why the removal of larger fibroids appears to have more beneficial effect than the removal of smaller fibroids. It can be hypothesised that the increasing size of fibroids increases the possibility and the extent of the potential mechanisms which result in subfertility, for example more extensive changes to local conditions such as vascular changes, hyperplasia, atrophy or inflammation of the endometrium [2].

Of the respondents who would intervene, they were less likely with increasing number of fibroids. The increasing tendency of surgeons not to intervene with increasing numbers of fibroids maybe related to the increasing likelihood of surgical complications, when more extensive and involved techniques are required to remove multiple fibroids. Indeed, as previously discussed, both laparoscopic and open myomectomy techniques have significant operative and postoperative morbidity especially with regards to areas of scarring which become non viable in terms of implantation.

\section{Conclusions}

In summary, there is a wide variation in current clinical practice in the UK in terms of the surgical management of fibroids which do not distort the uterine cavity and are associated with subfertility. If surgical intervention is favoured by the gynaecologist, patients must be well selected for the procedure based on the number, size and location of the fibroids. Allowing for the limitations of the research that has been published, the overall impression is that myomectomy has a role in the management of subfertility associated with fibroids. Myomectomy should not be withheld from any woman with fibroids experiencing subfertility and in whom no other aetiology for the subfertility is identified.

Acknowledgements We would like to thank Mrs. C. Allsopp for her administrative assistance.

Disclosure of interest None known.

Contribution to authorship GA: Responsible for the concept of the study. Involved in the design of the questionnaires and data analysis. Drafted the paper.

JD: Involved in the design of the questionnaires and data analysis. Drafted the paper.

YM: Involved in the design of the questionnaires and data analysis. Drafted the paper.

AW: Responsible for the concept of the study. Involved in the design of the questionnaires and data analysis. Drafted the paper. Supervised the study.

Details of ethics approval Not applicable.

Funding Not applicable. 


\section{References}

1. Ballerstones MA, Baroos DJC, Ochoa RS, Villalobos AS, Barroso VG, Sanchez SV, Gavino GF (2006) Effect of intramural and suberous myomas on in vitro fertilization cycles and their perinatal results. Ginecol Obstet Mex 74:55-65

2. Bajekal N, Li TC (2000) Fibroids, infertility and pregnancy wastage. Hum Reprod Update 6:614-620

3. Benecke C, Kruger TF, Siebert TI, Van der MErwe JP, Steyn DW (2005) Effects of fibroids on fertility in patients undergoing assisted reproduction. A structured literature review. Gynecol Obstet Investig 59:222-230
4. Dubuisson JB, Fauconnier A, Fourchotte V, Babaki-Fard K, Coste J, Chapron C (2001) Laparoscopic myomectomy: predicting the risk of conversion to an open procedure. Hum Reprod 16:17261731

5. Hurst BS, Matthews ML, Marshburn PB (2005) Laparoscopic myomectomy for symptomatic uterine myomas. Fertil Steril 83:1-23

6. Mukhopadhaya N, Asante GP, Manyonda IT (2007) Unterine fibroids: impact on fertility and pregnancy loss. Obstet Gynaecol Reprod Med 17(11):311-317

7. Stovall DW, Parrish SB, Van Voorhis BJ, Hahn SJ, Sparks AE, Syrop CH (1997) Uterine leiomyomas reduce the efficacy of assisted reproduction cycles: results of a matched follow-up study. Human Reprod 13:192-197 